\title{
SEO IN ACADEMIA?
}

\section{Autor-/Leser_innenschaft im Kontext algorithmischer Praktiken}

von JANA HERWIG

1 Roland Barthes: Der Tod des Autors [1968], in Fotis Jannidis u. a. (Hg.): Texte zur Theorie der Autorschaft, Stuttgart 2009, 185-193, hier 189 .
Seit Roland Barthes und Michel Foucault in den späten I96oer Jahren ihre folgenreichen Thesen zum «Tod des Autors» bzw. zur Autor_innenfunktion von Diskursen aufgestellt haben, haben sich die Praktiken des Schreibens und Publizierens im Kontext von Digitalisierung und Vernetzung erheblich gewandelt. Manch frühe Annahme hat sich dabei als Binsenweisheit erwiesen, etwa die, dass $<$ im Internet> alles von allen gesehen werde - praxeologisch ist es eher als glücklicher Umstand zu werten, wenn ins Netz gestellte Texte auch gefunden werden. Um die Wahrscheinlichkeit hierfür zu erhöhen, wenden Personen, die professionell online publizieren, Strategien der Textgestaltung und -aufbereitung an, die unter dem Kürzel SEO (search engine optimization) zusammengefasst werden. Diese sollen es dem dominierenden Typus der Crawler-basierten Suchmaschinen leichter machen, Inhalte zu finden und adäquat zu kategorisieren. Auch in der Wissenschaft ist diese Entwicklung mittlerweile zu spüren, begünstigt durch eine zunehmende Akzeptanz von Open-Access-Publishing, die steigende Nutzung von Online-Repositorien sowie den Marketing-Bestrebungen von Verlagen. Man kann diese Transformation aber auch anders beschreiben: als Erweiterung der Leser_innenschaft um nicht-menschliche Akteur_innen, nämlich die Programme der Suchmaschinen, die nun explizit miteinbezogen werden sollen. Wie sich Autor-/Leser_innenschaft im Kontext algorithmischer Praktiken reorganisiert, wird im Folgenden skizziert.

\section{Autor-/Leser_innenschaft reloaded}

Barthes' «Tod des Autors» entsprach letztlich der Einsetzung von Leser_innenschaft an Stelle von Autor_innenschaft. Dazu musste zunächst das geniegläubig-patriarchale Verständnis des «Autors» als eines «Vaters», der dem Werk zeitlich vorausgeht, für dieses «denkt, leidet, lebt», ${ }^{1}$ liquidiert werden. 
Die «wahre Schrift» in der Lektüre, die Barthes stattdessen postulierte, war zugleich noch immer menschlich definiert, wenn auch als «Mensch ohne Geschichte, ohne Biographie, ohne Psychologie», als «der femand, der in einem einzigen Feld alle Spuren vereinigt, aus denen sich das Geschriebene zusammensetzt $\gg .{ }^{2}$ Barthes' Beschreibung einer solchen biografiefreien, zusammensetzenden Leseinstanz, die zugleich Schrift ist, die «unentwegt Sinn» bildet, ${ }^{3}$ lässt sich nun überraschend gut übertragen auf die Vorgehensweise bestimmter Programme der Suchmaschinen, der sogenannten Crawler. Crawler sind per Programmierung damit beauftragt, im Web nach Content zu suchen, und treten dabei als nicht-menschliche Leser_innen in Erscheinung. Sie nehmen zunächst die Spur der Hyperlinks von Webseite zu Webseite auf, um so nach und nach in einer regelbasierten, algorithmischen Vorgehensweise das Web in seiner Gesamtheit zu erfassen. ${ }^{4}$ Gefundene Seiten werden von den Crawlern ausgelesen, dabei aber nicht im Volltext auf die Suchmaschinen-Server übertragen, sondern indexiert, also in Schlagwörtern und Auszügen in den Index übertragen und bewertet. Jeder menschlichen Lektüre von Texten, die über eine Suchmaschine gefunden werden, geht also eine zusammensetzende Lektüre durch Nicht-Menschen voraus, die zugleich Schrift bzw. Schreiben ist. Webseiten, die von keinem Crawler je in einen Index übertragen wurden, können schlicht mit keiner Suchmaschine gefunden werden. Diese Tätigkeit der Crawler, bei der diese Spuren vereinigen und das Geschriebene zusammensetzen, indem sie es neu einschreiben, ist somit der entscheidende Prozess, um Texte im Netz sichtbar zu machen - Autor-/Leser_innenschaft reloaded.

\section{Nach <dem Autor>: Textimmanenz}

Indem Foucault den «Autor» als Funktion bestimmte, mit der manche Diskurse ausgestattet seien, zeigte er auf, wie das «gewisse Vernunftwesen [...], das man Autor nennt», überhaupt erst konstruiert wird, ${ }^{5}$ bevor es als Deutungshorizont von Texten verwendet werden kann. Als zentral definiert Foucault dabei die «klassifikatorische Funktion» des Autor_innennamens, d.h. dessen Fähigkeit, Texte zu gruppieren, abzugrenzen, einige auszuschließen und sie anderen gegenüberzustellen. ${ }^{6}$ In den Lektüren bzw. Umschriften der Crawler sind Autor_innennamen jedoch vergleichsweise irrelevant: Stattdessen kreieren die Crawler ihren eigenen textimmanenten Deutungshorizont, indem sie die Häufigkeit und Position von Schlagwörtern im Text, Überschrift- und Absatzgestaltung, Bilderauszeichnungen, nur für den Crawler bereitgestellte Zusammenfassungen etc. heranziehen. ${ }^{7}$ Selbst die bei sekundärer Schulbildung mutmaßlich gewährleistete Lesbarkeit spielt eine Rolle bei der Bewertung der Relevanz eines Texts $^{8}$ - und hier wie bei allen herangezogenen Aspekten ist es eine Rolle unbestimmten Grades, da das präzise Verfahren der Crawler, ihr Algorithmus, nicht offenliegt. Um später gefunden zu werden, müssen Texte
2 Ebd., 192.

3 Ebd., 191.

4 Eine algorithmische Vorgehensweise, die z. B. Google mit menschlichem Suchverhalten vergleicht: "ähnlich wie du beim Surfen im Internet", Google LLC: Verarbeitung von Informationen in der GoogleSuche, in: Google - Suche o.J., google. com/intl/de/search/howsearchworks/ crawling-indexing (11.10.2020). 5 Michel Foucault: Was ist ein Autor?, in: Jannidis u. a.: Texte, 198-229, hier 214.

6 Ebd., 210.

7 Vgl. z. B. Bing.com: Bing Webmaster Guidelines, in: Microsoft Bing Webmaster Tools, o. J., bing.com/ webmaster/help/webmaster-guidelines3ofba23a (11.10.2020).

8 SEO-Professionals bewerten dies mithilfe des Flesch-ReadingEase-Tests, siehe dazu Anm. 12. 
9 An anderer Stelle zu untersuchen wäre, wie Suchmaschinen mit genderinklusiven Schreibweisen umgehen: Ob z. B. maskuline Formen vorgeschlagen werden, ob die Ergebnisseite Schreibweisen integriert etc.

10 Nicht berücksichtigt wird hier die Frage der Backlinks; von Koryphäen geschriebene Texte könnten z. B. im Verlauf der Zeit reputationsfördernde Backlinks auf sich selbst akkumulieren.

11 Rückmeldung des Tools Yoast SEO auf eine Zwischenfassung des vorliegenden Texts.

12 So eine weitere Rückmeldung von Yoast SEO. Der Flesch-Index basiert nicht auf semantischer Analyse, sondern auf dem Verhältnis von Silbenanzahl pro Wort und durchschnittlicher Satzlänge, vgl. Peter Schoell: Die Flesch-Formel, in: Fleschindex berechnen, o.J., fleschindex. delformel (11.10.2020).

13 Rückmeldung von Yoast SEO.

14 Wiley Authors: Search Engine Optimization (SEO) for your article, in: Wiley Author Resources, o.J., authorservices.wiley.com/author-resour ces/Journal-Authors/Prepare/writing-forseo.html (11.10.2010). nicht durch ausgewiesene Autor_innenschaft, sondern durch sich selbst einlösen, was sie durch sich selbst versprechen: Ein Text zum Thema Autor_innenschaft, der das Wort <Autor_innenschaft> kaum, nicht an den richtigen Stellen oder in für den Crawler nicht lesbarer Form (z. B. als Bild) ${ }^{9}$ verwendet, wird für dieses Suchwort kaum hoch gerankt werden - unabhängig davon, von wem er verfasst wurde. ${ }^{10}$

\section{Assistiertes Schreiben}

Wenn aber Texte, um gefunden zu werden, durch sich selbst einlösen müssen, was sie versprechen, dann müssen Texte von Grund auf anders geschrieben werden. Schreibende müssen dazu die Fähigkeit entwickeln, leicht lesbare Texte $\mathrm{zu}$ verfassen und an den richtigen Stellen mit geeigneten Schlüsselbegriffen zu versehen. Content-Management-Systeme stellen dafür Tools zur Verfügung, die schon im Schreibprozess selbst nach jedem Abspeichern Hinweise geben, wie ein Text SEO-technisch verbessert werden kann, basierend auf rein textstatistischen statt semantischen Auswertungen. «Das Fokus-Keyword scheint im ersten Absatz des Textes nicht vorzukommen. Stelle umgehend sicher, dass das Thema klar ist.»"11 Jene Schreibenden, die sich bislang als allein über ihren Text verfügende Instanz wähnten, müssen nun dabei lernen, sich auf die Ermahnungen dieser nicht-menschlichen Assistent_innen einzulassen, die - als lokale Crawler-Simulatoren - fortwährend das Ergebnis ihrer Probelektüren mitteilen. «Der Text erzielt 3 I.9 in dem Flesch Reading-Ease Test, was als schwer zum Lesen betrachtet wird. Versuche kürzere Sätze zu formulieren, und weniger komplizierte Wörter zu benutzen, um die Lesbarkeit zu erhöhen.» ${ }^{12}$ Der Anrufung der Schreibenden als gute Kommunikator_innen liegen dabei generalisierende, statistische Urteile zugrunde, die anzunehmen nicht immer leicht fällt. «Nur 6.3\% der Sätze enthalten Bindewörter. Das reicht nicht aus. Verwende mehr davon. ${ }^{13}$

\section{SEO in Academia?}

Auch Verlage haben den möglichen Beitrag der Autor_innen zur SEO-Optimierung bereits für sich entdeckt. So bieten etwa die Wiley Author Services Anleitungen zum konsistenten Einsatz von Keywords und empfehlen obendrein, geeignete Schlagwörter mithilfe von SEO-Marketing-Tools zu identifizieren: «Remember that keywords are important for A\&I services as well as SEO.» ${ }^{14} \mathrm{~A} \&$ I steht für <Abstracting and Indexing>, also für die Erstellung von Abstracts und Indizes als bibliografischen Hilfsmitteln. Denn selbstverständlich sind Praktiken der Indexierung in der Wissenschaft schon lange etabliert. Im Kontext digital-vernetzter Praktiken verschiebt sich deren Ausrichtung jedoch derzeit: weg von einer Dienstleistung, die am finalen Text ansetzt, hin zu einer Empfehlung, die in den Schreibprozess selbst eingegliedert werden 
möchte und dabei an das Eigeninteresse der Autor_innen appelliert: «You», so Wiley, «can also play a crucial role in optimizing the search results for your article - helping people to find, read, and cite your work». ${ }^{15}$ Deutschsprachige Verlage arbeiten hier vor allem mit der nachgelagerten Produktion von Epitexten verschiedener Länge (z.B. Blurbs, Headlines, Schlagworten ${ }^{16}$ ), die sich freilich jetzt schon typischen SEO-Texten zuordnen lassen (z.B. SERP Snippet, Heading, Keywords' ${ }^{17}$. Das akademisch schreibende Selbst soll auch ein unternehmerisches Selbst sein, das seinen Text von der (Keyword-)Recherche für den Markt der Aufmerksamkeit aufbereitet. Ob eine höhere Sichtbarkeit dank SEO auch wissenschaftliche Karrieren fördert oder bei der Bewältigung gesellschaftlicher Herausforderungen im Sinne der Third Mission dienlich sein kann, wird letztlich eine Frage der Kongruenz oder Divergenz von Wissenschaft, Wirtschaft und Gesellschaft sein.
15 Ebd.

16 Das ist z. B., neben Abstracts, die verwendete Klassifizierung von Kurztexten bei Nomos Baden.

17 SERP = Search Engine Results Page. Snippets sind Inhaltszusammenfassungen, die als Textvorschau auf der SERP angezeigt werden. 